\title{
Understanding and strain-engineering wrinkle networks in supported graphene through simulations
}

\author{
Kuan Zhang, Marino Arroyo* \\ Laboratori de Càlcul Numèric (LaCàN), Universitat Politècnica de Catalunya (UPC), \\ Campus Nord UPC-C2, E-08034 Barcelona, Spain
}

\begin{abstract}
Wrinkle networks are ubiquitous buckle-induced delaminations in supported graphene, which locally modify the electronic structure and degrade device performance. Although the strong property-deformation coupling of graphene can be potentially harnessed by strain engineering, it has not been possible to precisely control the geometry of wrinkle networks. Through numerical simulations based on an atomistically informed continuum theory, we understand how strain anisotropy, adhesion and friction govern spontaneous wrinkling. We then propose a strategy to control the location of wrinkles through patterns of weaker adhesion. This strategy is deceptively simple, and can in fact fail in several ways, particularly under biaxial compression. However, within bounds set by the physics of wrinkling, it is possible to robustly create by strain a variety of wrinkle network geometries and junction configurations. Graphene is nearly unstrained in the planar regions bounded by wrinkles, highly curved along wrinkles, and highly stretched and curved at junctions, which can either locally attenuate or amplify the applied strain depending on their configuration. These mechanically self-assembled networks are stable under the pressure produced by an enclosed fluid and form continuous channels, opening the door to nano-fluidic applications.
\end{abstract}

Keywords: graphene, wrinkles, blisters, strain engineering, buckling

Email address: marino.arroyo@upc.edu (Marino Arroyo*) 


\section{Introduction}

Rather than a flatland, supported graphene describes a landscape shaped by out-of-plane features with different physical origins. Defects such as dislocations or grain boundaries can relax through out-of-plane deformations (Liu and Yakobson, 2010; Yakobson and Ding, 2011; Zhang et al., 2014), which have been observed experimentally (Duong et al., 2012; Warner et al., 2013). Gas trapped between graphene and the substrate, either unintentionally (Stolyarova et a1., 2009; Georgiou et a1., 2011) or in a controlled manner (Bunch et al., 2008; Koenig et al., 2011; Zabel et al., 2012; Pan et al., 2012; Kitt et al., 2013), can elastically deform graphene, producing blisters of various shapes and sizes. Lateral strain produced upon cooling of graphene grown by chemical vapor deposition (CVD) on solid metallic substrates invariably results in linear and localized wrinkles (Li et al., 2009; Robertson et al., 2011; Obraztsov et al., 2007; Zhu et al., 2012; Liu et al., 2012), which

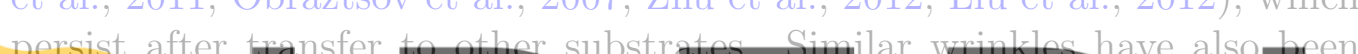
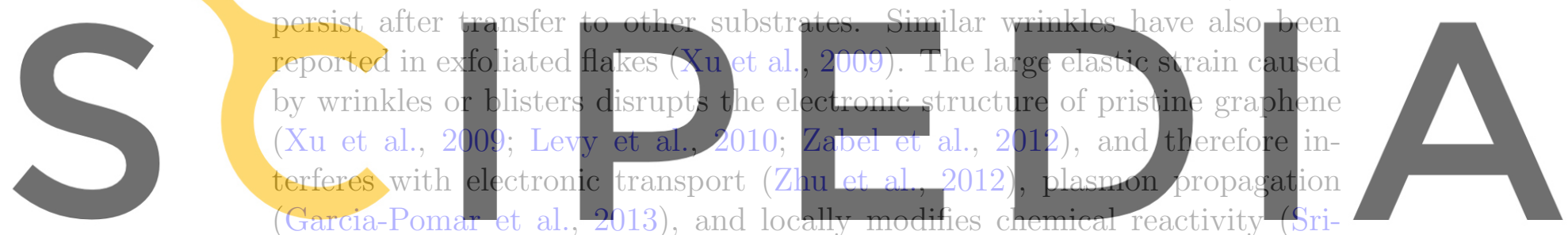

vastava et al., 1999; Ruoff, 2012). For this reason, blisters and wrinkles are

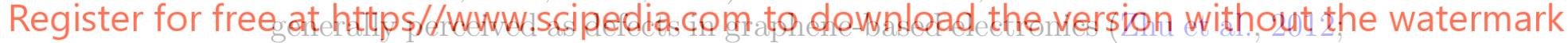

Jiang et al., 2013) and optics (Garcia-Pomar et al., 2013). It has also been suggested that the strong coupling between localized deformation and electronic structure could be exploited by strain engineering(Levy et al., 2010; Castellanos-Gomez et al., 2013; Ruoff, 2012; Pereira et al., 2010; Zabel et al., 2012).

Here, we focus on wrinkles in supported graphene, generally understood as buckle-induced delaminations (Vella et al., 2009) caused by lateral compression. By intentionally straining the substrate uniaxially, it has been shown that lateral strain is transmitted to graphene by frictional forces, leading to localized wrinkling of monolayer graphene perpendicular to the strain direction (Jiang et al., 2013). Theoretical calculations suggest that, upon uniaxial compression, the localized wrinkles in graphene are preceded by distributed ripples of very small amplitude (Zhang and Arroyo, 2013), see Fig. 1(a) Most wrinkles observed in CVD graphene form two-dimensional networks (Li et al., 2009; Robertson et al., 2011; Obraztsov et al., 2007; Zhu 
et al., 2012; Liu et al., 2012), indicative of biaxial compressive strain and suggestive of stress focusing (Cerda et al., 1999; Witten, 2007; Pereira et al., 2010; Aoyanagi et al., 2010). Beyond isolated wrinkles, massive crumpling and delamination has been reported in supported multilayer graphene under very large biaxial compression (Zang et al., 2013).

A number of experimental strategies have attempted to control wrinkle networks in graphene. It has been shown that the transfer process can increase, decrease, or even eliminate wrinkling (Liu et al., 2011; Calado et al., 2012), and that wrinkles preferentially form at topographical features of the substrate (Kim et al., 2011a; Pan et al., 2011; Liu et al., 2012). However, it has not been possible to precisely and reversibly control the location of wrinkles, partly due to an insufficient theoretical understanding of wrinkling under biaxial compression (Zhu et al., 2012). Here, through detailed simulations (Section 2), we examine the spontaneous formation of wrinkle networks under biaxial strain (Section 3), identify the key governing mechanisms including
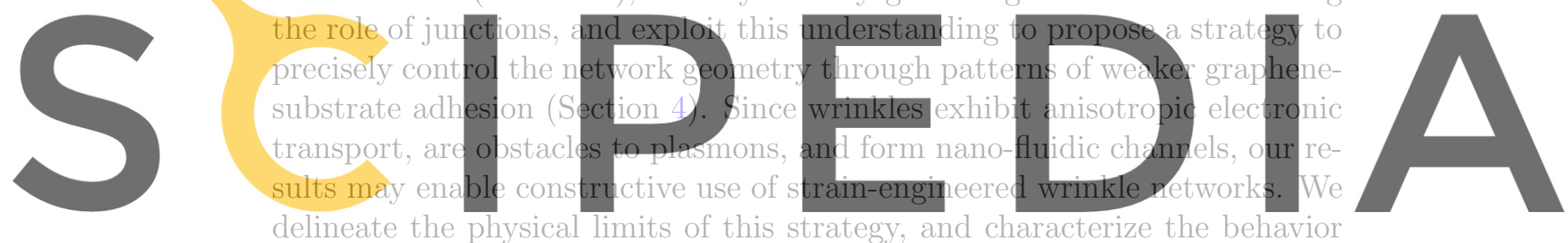

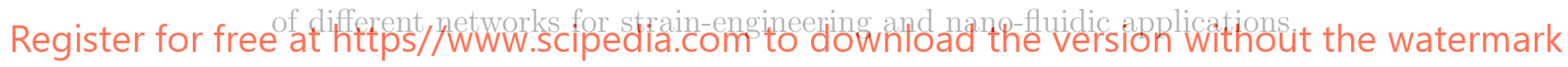

\section{Model and simulation method}

We consider a mesoscopic $\left(500 \times 500 \mathrm{~nm}^{2}\right)$ graphene sample supported on a substrate undergoing lateral deformation under periodic boundary conditions, see Fig. 1(b). The domain is large enough to capture the typical wrinkle separation (several $100 \mathrm{~nm}$ ) but small enough to be computationally tractable, since the geometric features of an individual wrinkle $(\sim 1 \mathrm{~nm})$ (Zhu et al., 2012; Liu et al., 2012) need to be resolved.

\subsection{Model for graphene}

We model graphene with an atomistic-based continuum theory, built from Brenner's force field and discretized with subdivision finite elements (Arroyo and Belytschko, 2002, 2004b). This method has been shown to accurately simulate carbon nanotubes (Arias and Arroyo, 2008) and graphene 
(Zhang and Arroyo, 2013; Ghosh and Arroyo, 2013) undergoing large deformations and buckling instabilities at a fraction of the computational cost of an atomistic model. In this continuum model, we describe the configuration of graphene by a surface parametrization $\boldsymbol{x}(u, v)=(x(u, v), y(u, v), z(u, v))$, where $z(u, v)$ is the coordinate perpendicular to the substrate. The elasticity of this continuum description of graphene is modeled through a hyperelastic potential of the form $W(\boldsymbol{C}, \mathcal{K})$ that depends on the in-plane strain (the Cauchy-Green deformation tensor) of the surface $\boldsymbol{C}$ and its curvature (the second fundamental form) $\mathcal{K}$. The energy density $W$ is systematically derived from the atomistic model describing the bonding energy and forces using a kinematic rule that links continuum and lattice deformations, the exponential Cauchy-Born rule, and averaging the atomistic energy in one unit cell of the lattice. The total elastic energy of the graphene sheet is then


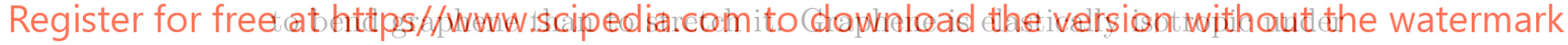

infinitesimal deformations (Arroyo and Belytschko, 2004a), and only becomes noticeably anisotropic when severely strained (Lu et al., 2009). Our model is geometrically exact and accounts for strain-induced anisotropy, but we found that our simulations are insensitive to the lattice orientation relative to the domain and pattern geometry.

\subsection{Applying strain}

We apply strain by progressively reducing the lateral dimensions of the periodic simulation box. The strain is viewed as externally applied by deforming the substrate, although it can occur from differential thermal expansion, as in graphene synthesis by CVD. We denote by $\lambda_{x}$ and $\lambda_{y}$ the stretch ratios between the current and initial lateral dimension along each coordinate, and by $\varepsilon_{c}=1-\lambda_{x} \lambda_{y}$ the compressive areal strain (normalized area difference). Implicit in these boundary conditions is the assumption that the deformation of the substrate uniformly conforms to the externally applied strain, i.e. its 


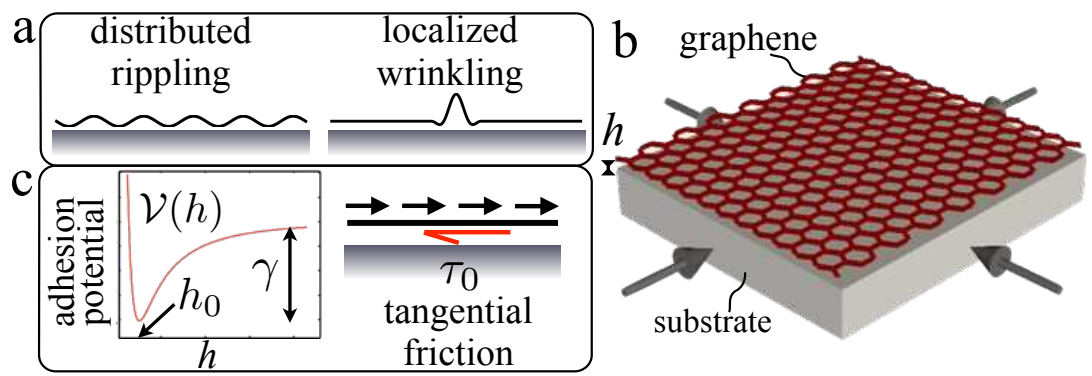

Figure 1: (a) Upon compression, confined thin films develop a variety of out-of-plane deformations, classified here as rippling, distributed wave-like disturbances, or wrinkling, localized out-of-plane features surrounded by planar regions. (b) Model setup for a graphene flake adhered to a substrate and laterally compressed. $h$ is the graphene-substrate separation. (c) Graphene interacts with the substrate through an adhesion potential $\mathcal{V}(h)$, Aitken and Huang (2010) characterized by the adhesion energy $\gamma$ and the equilibrium separation $h_{0}$, and through tangential forces opposing sliding, modeled as dry friction with interfacial shear strength $\tau_{0}$ (Zhang and Arroyo, 2013).
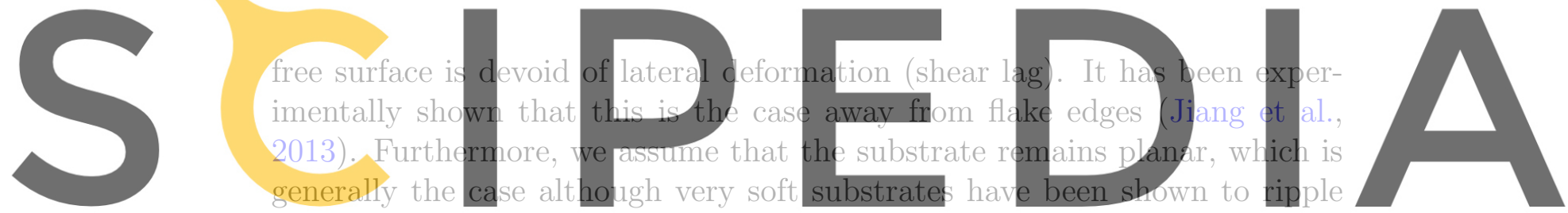

along with graphene (Wang et al., 2011).

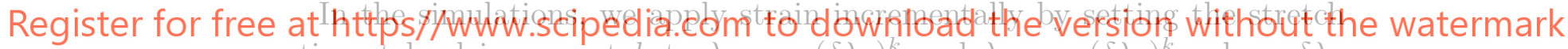

ratios at load increment $k$ to $\lambda_{x, k}=\left(\delta \lambda_{x}\right)^{k}$ and $\lambda_{y, k}=\left(\delta \lambda_{y}\right)^{k}$, where $\delta \lambda_{x}$

denotes the incremental stretch ratio along $x$. To obtain the equilibrium configuration at step $k+1$, we modify the previous equilibrium configuration step $\boldsymbol{x}_{k}(u, v)$ following $\left(\delta \lambda_{x} x_{k}(u, v), \delta \lambda_{y} y_{k}(u, v), z_{k}(u, v)\right)$ to define an initial guess in the iterative procedure consistent with the boundary conditions.

\subsection{Model for the graphene-substrate interaction: adhesion and friction}

Graphene interacts with the substrate non-covalently, resulting in perpendicular adhesive forces and tangential frictional forces opposing sliding, see Fig. 1(c). The non-bonded adhesion energy density per unit surface $\mathcal{V}(h)$ is given by an effective 3-9 Lennard-Jones potential between a point in graphene and a half-space (Aitken and Huang, 2010; Zhang and Arroyo, 2013)

$$
\mathcal{V}(h)=-\gamma\left[\frac{3}{2}\left(\frac{h_{0}}{h}\right)^{3}-\frac{1}{2}\left(\frac{h_{0}}{h}\right)^{9}\right],
$$


where $\gamma$ is the adhesion energy and $h_{0}$ the equilibrium separation. The resulting total adhesion energy takes the form

$$
U_{a d h}[\boldsymbol{x}]=\int_{\Omega_{0}} \mathcal{V}(z) d S_{0}
$$

The material parameters determining the graphene-substrate interaction have been shown to vary significantly, and for this reason we consider a range of parameter values consistent with experimental measurements. We did not find the behavior of the system to be qualitatively sensitive to changes in the the adhesion energy within a realistic range. We adopt here $\gamma=0.45 \mathrm{~J} / \mathrm{m}^{2}$, typical of the interaction between graphene and $\mathrm{SiO}_{2}$ (Koenig et al., 2011), half of than that reported for copper (Yoon et al., 2012), but a few times larger than that on polydimethylsiloxane (Scharfenberg et al., 2012). As for the equilibrium separation $h_{0}$, which controls the decohesion separation and also the stiffness of this interaction, we range from $0.5 \mathrm{~nm}$, in the order of that measured on $\mathrm{SiO}_{2}$ (Gupta et al., 2006) to $6 \mathrm{~nm}$, comparable to that re-
ported for a polymeric substrate (Wang et al., 2011). Such large equilibrium
separations should be interpreted as an fffective property for substrates that
are not atomically flat. Substrate roughness has been shown to modify the 4 roughness is comparable to the equilibrium separation (Aitken and Huang, Register for freelathttes/Awwiw:scipedia).com to download the version without the watermark

We model the stripes of weaker adhesion by spatially modulating the adhesion energy as $\gamma(x, y)=\bar{\gamma}\left\{1-\exp \left[-\operatorname{dist}(x, y ; \ell)^{2} / c^{2}\right]\right\}$, where $\operatorname{dist}(x, y ; \ell)$ denotes the distance of a point to the closest line in the pattern $\ell$. We take $c=1 \mathrm{~nm}$, which leads to a stripe width of $\sim 4 \mathrm{~nm}$. We found that the results are quite insensitive to doubling the width. However, if the stripe width becomes comparable to $\sqrt{D / \gamma} \approx 0.7 \mathrm{~nm}$, then bending stiffness can significantly impede the formation of wrinkles conforming to such narrow stripes.

We model the tangential forces as dry friction (Zhang and Arroyo, 2013). Since dry friction results in a non-smooth model, we consider a regularized model of friction for numerical convenience with an incremental work of friction of the form

$$
U_{f r, k}[\boldsymbol{x}]=\int_{\Omega_{0}} \tau_{0} K\left(s_{k}(u, v)\right) d S_{0},
$$

where $\tau_{0}$ is the interfacial shear strength, $s_{k}$ is the incremental lateral sliding 
relative to the substrate

$$
s_{k}^{2}(u, v)=\left[x-\delta \lambda_{x} x_{k}(u, v)\right]^{2}+\left[y-\delta \lambda_{y} y_{k}(u, v)\right]^{2},
$$

and $K(t)$ is a differentiable approximation to $|t|$. Here, we consider the $\log$ sum-exp regularization

$$
K(t)=\frac{1}{\beta} \log \left(e^{\beta t}+e^{-\beta t}\right)
$$

for $\beta>0$. As $\beta \rightarrow+\infty$, it can be shown that this family of smooth functions converges to the absolute value function. In our calculations, we choose $\beta=200 \mathrm{~nm}^{-1}$. The quantity $1 / \beta=1 / 200 \mathrm{~nm}$ can be interpreted as the length-scale over which the cusp of the absolute value around zero is being rounded, and therefore for sliding displacements larger than $1 / \beta, K(t)$ is indistinguishable from $|t|$ and we recover dry friction. We have checked
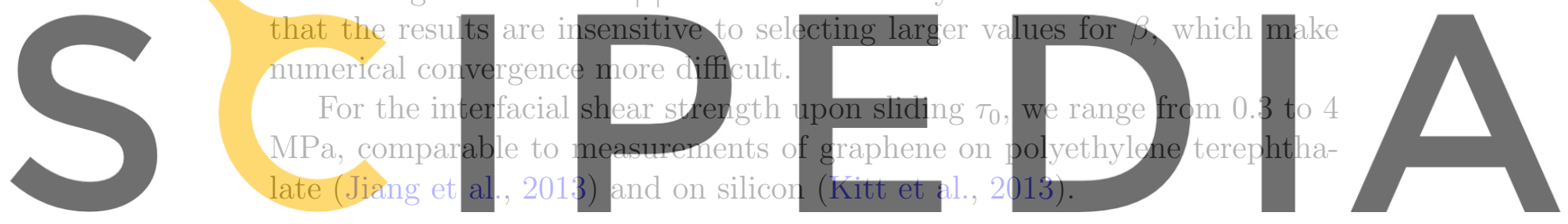

Register for free at https//WwW. Scipedia.com to download the version without the watermark

$$
U_{e l}[\boldsymbol{x}]+U_{a d h}[\boldsymbol{x}]+U_{f r, k}[\boldsymbol{x}]
$$

is discretized with subdivision finite elements, and at each strain increment, we obtain stable equilibrium configurations by numerical minimization using a quasi-Newton method combined with line-search (Arroyo and Belytschko, 2004b). The $500 \mathrm{~nm} \times 500 \mathrm{~nm}$ simulation box is subdivided into finite elements, resulting in $3 \cdot 10^{6}$ degrees of freedom. For simulations with the honeycomb pattern, the periodic simulation box is $433 \mathrm{~nm} \times 375 \mathrm{~nm}$.

Rippling and wrinkling are geometric instabilities that break the symmetry of the initially uniform system. Therefore, one expects a massive metastability and non-uniqueness of equilibrium configurations in the real system, and also in the numerical solution. Both the physical system and the numerical model should not be expected to attain an absolute energy minimizer at all times due to energy barriers. As a result, simulations with 


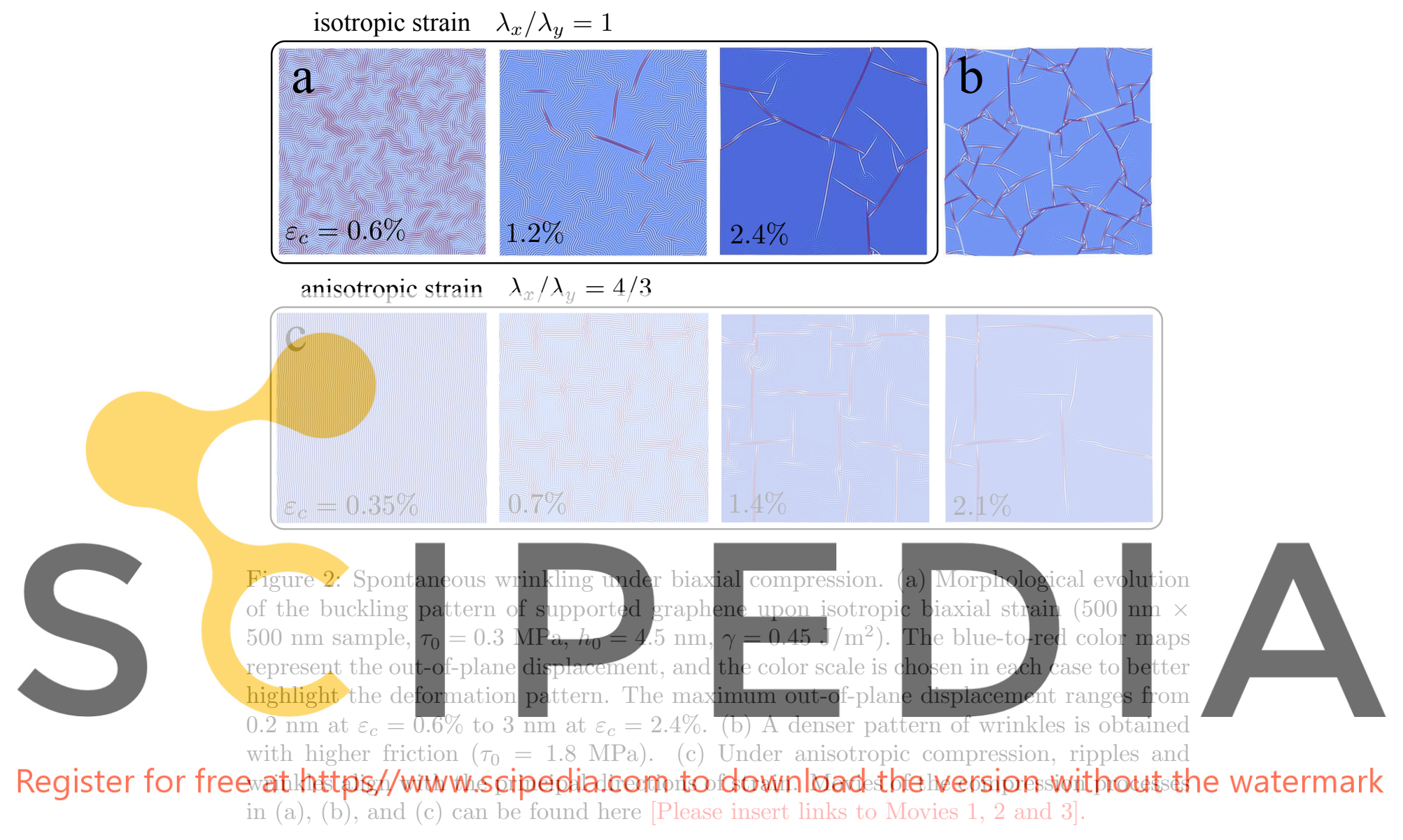

identical physical parameters but slight differences in the numerical parameters can produce wrinkle patterns of very different geometry. Despite this generic and unsettling feature of buckling in highly symmetric systems, simulations provide robust information about global characteristics of wrinkling, such as the wrinkle density, alignment, or the behavior of the different energy components as a function of strain. These issues and the verification of the computer algorithms are examined in Appendix A.

\section{Spontaneous wrinkling}

We first examine the spontaneous emergence of localized wrinkles upon isotropic compression, see Fig. 2(a). Beyond a threshold strain, the system departs from a planar state and develops a distributed, small-amplitude rip- 
pling instability throughout the sample $\left(\varepsilon_{c}=0.6 \%\right)$, but since it produces polarized plane-wave deformations and strain is isotropic, many domains with random orientations develop, similar to labyrinth patterns previously reported in other compressed thin films (Huang et al., 2005). Upon further compression $\left(\varepsilon_{c}=1.2 \%\right)$, the out-of-plane deformation localizes into short wrinkles, which partially release rippling in their vicinity. Localization is caused by the decohesion of the graphene-substrate interaction. Ripple and wrinkle coexistence has been reported in CVD graphene samples (Liu et al., 2012). As compressive strain increases, wrinkles grow and connect to form a network partitioning the sample into nearly flat subdomains. During this reorganization, most ripples and some wrinkles disappear, and the network simplifies by aligning and merging neighboring wrinkles that were previously along similar directions $\left(\varepsilon_{c}=2.4 \%\right)$. While wrinkles can form complex structures where they meet, the abundance of simple T-junctions is noteworthy. Once the wrinkle network has matured, further compression increases the wrinkle height without signifiont reorganization. The equitibrium separation chosen here is relatively large to better visualize the competition between
distributed rippling and localized wrinkling, which results in a compliant in-
teraction since the stifness of the adhesion potential is proportonal to $\gamma / h_{0}^{2}$.
For very tight interaction as in atomically flat substrates, e.g. ho below $1 \mathrm{~nm}$,

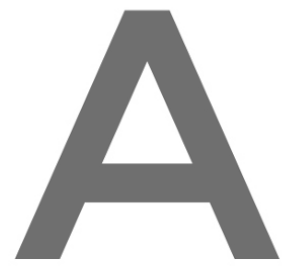

the system withstands larger strains before buckling and then abruptly tranthe watermark

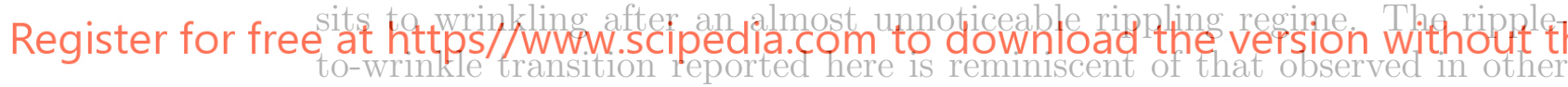
elastic sheets coupled to different substrates (Pocivavsek et al., 2008; Zang et al., 2012; Kim et al., 2011b).

Spontaneous wrinkling can be modulated in various ways. Since the emergence of wrinkling requires significant sliding of graphene relative to the substrate, frictional forces determine the expected separation between wrinkles (Zhang and Arroyo, 2013). By considering a significantly larger interfacial shear strength in Fig. 2(b), many more short wrinkles nucleate and the reorganization of the wrinkle network is hindered, leading to smaller subdomains. Friction could be modulated experimentally by controlling the pressure difference across the graphene sheet (Kitt et al., 2013; Pugno et al., 2013), since graphene is impermeable to common gases (Bunch et al., 2008). On the other hand, strain anisotropy leads to ripples and wrinkles aligned with the principal directions of anisotropy (Huang et al., 2005; Kim et al., 2011b), see Fig. 2(c). The similarity between the isotropic (Li et al., 2009; Robertson et al., 2011; Obraztsov et al., 2007; Calado et al., 2012; Liu et al., 


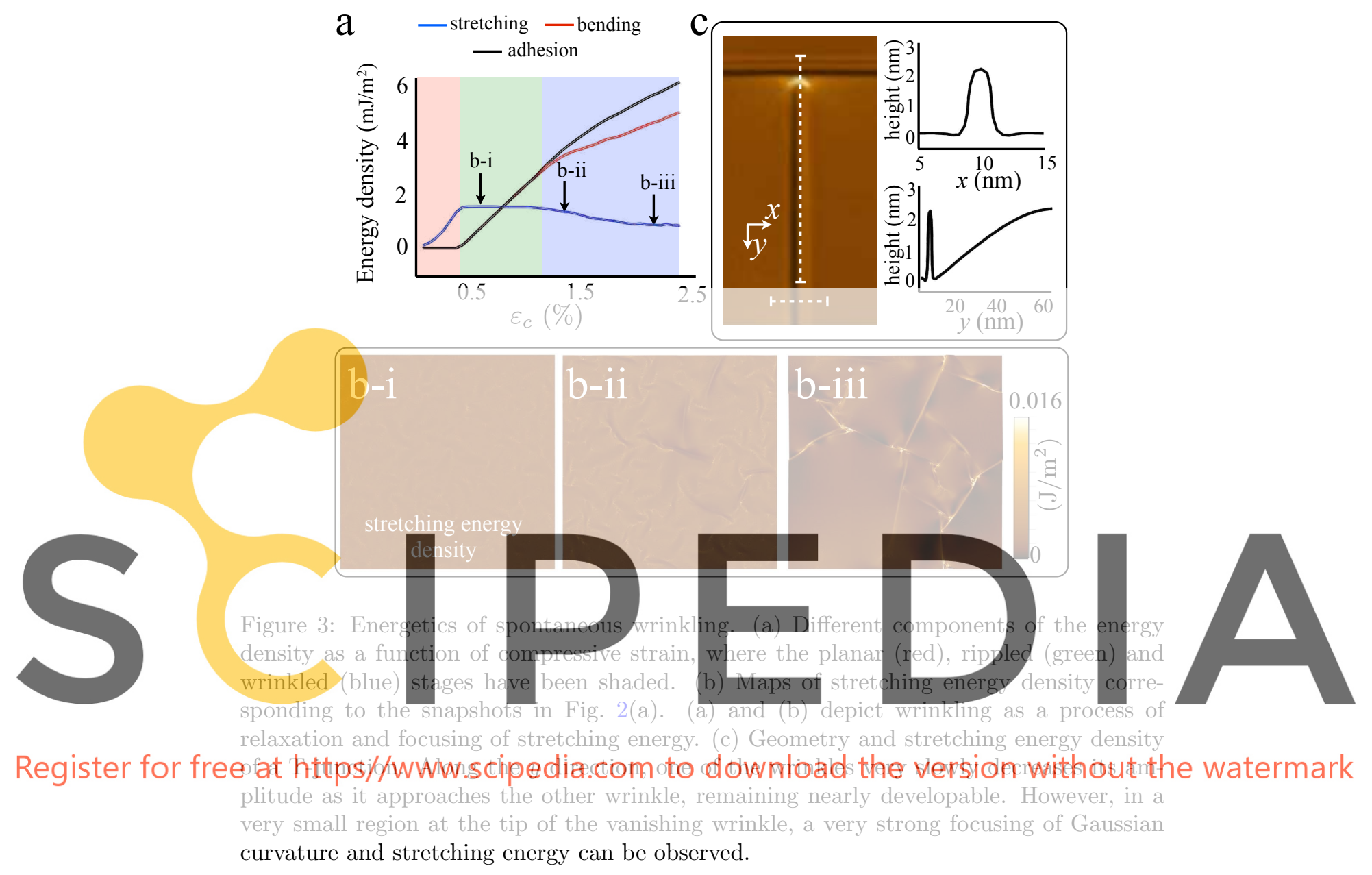

2012) and anisotropic (Zhu et al., 2012; Liu et al., 2012) wrinkle patterns observed in supported single and few-layer graphene and those obtained here is remarkable.

To better understand the mechanics leading to these wrinkle networks, we analyze next the energetics of the process. Fig. 3(a) shows different components of the energy (stretching, bending and adhesion) per unit area as a function of compressive strain. While the sample remains planar and at its equilibrium separation (red-shaded region), the stretching energy is the only contribution, and it grows quadratically as expected for an elastic material under small deformation. At a critical strain, it is energetically favorable to further accommodate compressive strain by distributed rippling. This out-of- 
plane deformation upsets the bending and adhesion energies, which linearly increase, but keeps stretching energy constant (green-shaded region). The competition between adhesion and bending energy sets the rippling wavelength. The figure shows that, beyond a second threshold, localized wrinkling further relaxes the energy; bending and adhesion energies grow at a slower rate, and stretching energy progressively reduces. This response under biaxial compression is very different from the wrinkling behavior under uniaxial compression, where stretching energy reduces towards zero much faster. Here, it is not possible to accommodate biaxial compression without significant stretching, which becomes a key factor in understanding wrinkle networks and the junctions they form.

Unlike bending deformations, in-plane stretching cannot be directly visualized from the out-of-plane displacement maps. For this reason, we show in Fig. 3(b) the spatial distributions of stretching energy during the process. While in the rippling stage (b-i) the stretching energy is quite uniformly distributed, it progressively relaxes around the nascent wrinkles (b-ii). When the wrinkle network is fully formed (b-iii), the contrast in stretching energy distribution dramatically increases, by nearly relaxing in most of the sample and strongly focusing it along some wrinkles and mainly at wrinkle junctions and other network point defects. Thus, wrinkling can be understood as a process of stretching energy relaxation and focusing, at the expense of bending and adhesion energy.

The relation between sheet morphology and stretching can be understood from Gauss Theorema Egregium, which implies that doubly-curved (nondevelopable) regions of the film are necessarily stretched, while developable straight wrinkles can exist without stretching (Witten, 2007). Junctions involve localized double curvature, which explains why stretching focuses at points, while bending and decohesion also concentrate along the linear singly-curved wrinkles. Amongst the diversity of junction morphologies, Tjunctions appear to be stable low-energy configurations, see Fig. 3(c). Interestingly, T-junctions are frequently observed in supported graphene (Liu et al., 2012). In the context of crumpling of unsupported thin elastic sheets including graphene, focusing of stretching energy at point (developable cones) and line (stretching ridges) deformation features has been extensively studied (Cerda et al., 1999; Witten, 2007; Pereira et al., 2010). T-junctions between wrinkles seem to be an analogous building block for compressed and adhered sheets (Aoyanagi et al., 2010). 

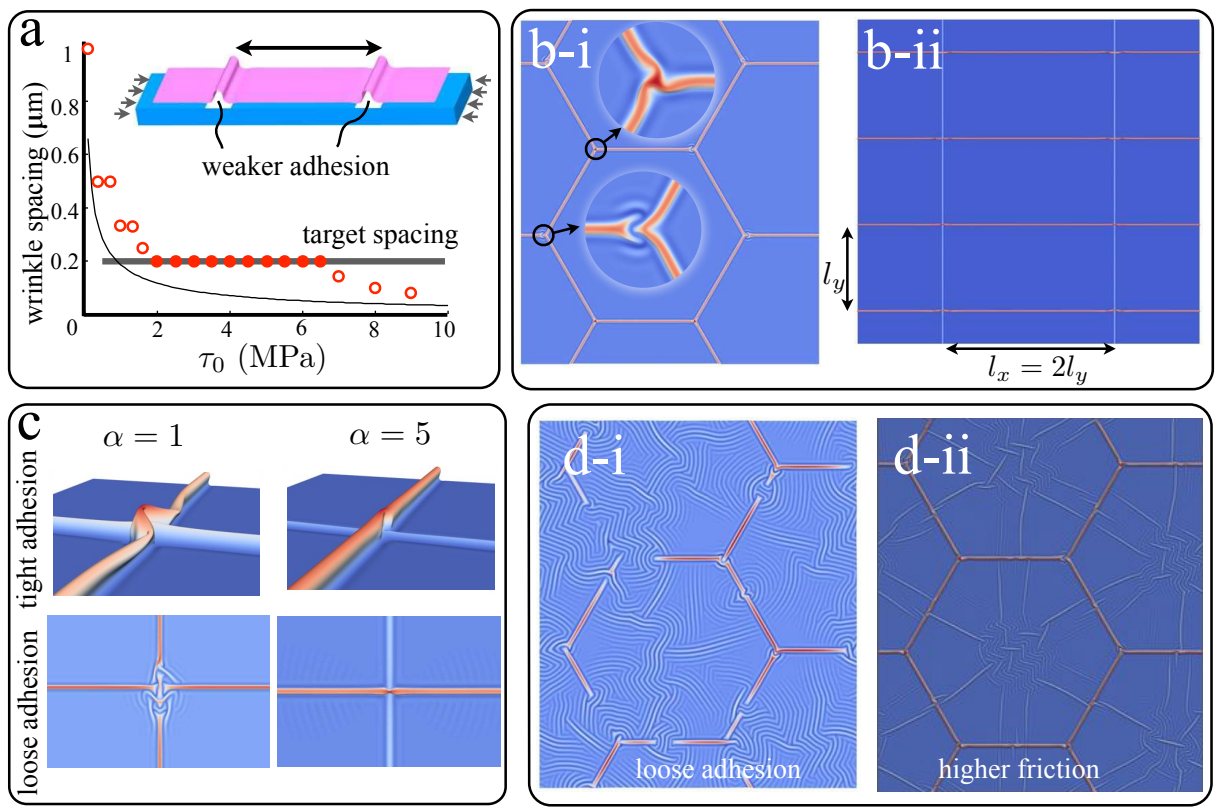

Figure 4: Controlling the wrinkle location with stripes of lower adhesion. Under uniaxial compression, (a) shows the average wrinkle spacing as a function of $\tau_{0}$ (red circles), compared to the target spacing (horizontal gray line) set by the separation between stripes. Simulations where wrinkles conform to the stripes are shown as filled red circles. For very large $\tau_{0}$, additional wrinkles form between stripes, while for very small $\tau_{0}$ wrinkles do not form in every stripe. The black curve shows the theoretical wrinkle separation as a function of $\tau_{0}$ in the absence of stripes. This idea can be extended to two-dimensional wrinkle networks under isotropic (b-i) and anisotropic (b-ii) biaxial loading, if the adhesion is tight enough (here, $h_{0}=1.5 \mathrm{~nm}$ and $\tau_{0}=4 \mathrm{MPa}$ ). $\alpha=l_{x} \lambda_{x} /\left(l_{y} \lambda_{y}\right)$ measures the nominal height ratio between the two sets of wrinkles in the rectangular pattern. As shown in (c), X-junctions become unstable if the nominal height ratio is close to one, by either kinking for tight adhesion or by spreading into a rippled pattern for loose adhesion. $\mathrm{X}$-junctions are stabilized if the nominal height of the kinks is significantly different. (d) shows how the honeycomb pattern fails to form if the adhesion is too loose (d-i) or the friction too high (d-ii). 


\section{Controlling wrinkle networks}

\subsection{Using patterns of stripes of weak adhesion}

With this understanding of the mechanics of spontaneous wrinkling, we turn now to strategies to precisely control the geometry of the wrinkle network amongst the vast range of available wrinkling pathways. While controlling buckling instabilities is notoriously difficult, particularly under multiaxial compression, there have been different attempts to guide wrinkles in different supported thin films (Hendricks et al., 2010). Wrinkling patterns in metal-capped thin polymer films have been modulated, but not precisely controlled, by chemically patterning the adhesive properties of the substrate (Vandeparre et al., 2007). Wrinkles in supported graphene have been shown to predominantly form at substrate corrugations under uniaxial compression (Kim et al., 2011a; Pan et al., 2011; Liu et al., 2012). Building on these ideas, we attempt to conform wrinkles to patterns of stripes of weaker adhesion in an otherwise homogeneous substrate. Such patterns may be implemented through physicochemical modification and nanolithography. We examine first uniaxial compression, where in the absence of stripes the wrinkle separation strongly depends on the frictional properties (Zhang and Arroyo, 2013), see the black curve in Fig. 4(a). We expect that the frictional selection principle for wrinkle separation may interfere with our strategy to control the wrinkle location, e.g. uncontrolled wrinkles could form between the stripes for very high friction or some stripes may fail to accommodate a wrinkle for very small friction. To address this question, we uniaxially compress a $1 \mu \mathrm{m}$ graphene ribbon supported on a substrate decorated with uniformly spaced stripes $4 \mathrm{~nm}$ wide and $200 \mathrm{~nm}$ apart. Fig. 4(a) shows the average wrinkle spacing (red circles) obtained from simulations with different frictional properties. It can be observed that, given a target wrinkle separation, wrinkles conform to stripes in a relatively wide range of interfacial shear strength. Thus, stripes of weak adhesion appear to be a robust method to control wrinkle location if properly tuned to the graphene-substrate friction.

We extend next this idea to two-dimensional isotropic and anisotropic stripe patterns under biaxial loading, see Fig. 4(b). For isotropic compression, we attempt an isotropic honeycomb pattern of stripes, and observe that wrinkles can conform to the pattern, forming two types of Y-junctions with broken-symmetry, Fig. 4(b-i). Since under anisotropic compression we found that spontaneous wrinkles form along perpendicular directions, we choose a rectangular grid of stripes. We find that wrinkles first form perpendicu- 
lar to the direction of maximum strain, and then match the target pattern as a second family of wrinkles develops, forming $\mathrm{X}$-junctions with the first family, Fig. 4(b-ii). The wrinkle networks robustly conform to the desired patterns for a wide range of parameter values, but the proposed strategy fails if the pattern is not properly tuned the adhesive/frictional properties, or to the compression anisotropy. For instance, X-junctions can become unstable, Fig. 4(c), particularly when the two families of perpendicular wrinkles have the same nominal height $(\alpha=1)$. For tight adhesive interactions, e.g. $h_{0}=0.5 \mathrm{~nm}$ representative of graphene on an atomically flat surface, $\mathrm{X}$-junctions become severely strained and kink. For very loose interactions, e.g. $h_{0}=6 \mathrm{~nm}$, which may model a soft polymeric substrate, X-junctions destabilize into a complex partially rippled structure. X-junctions can be stabilized by changing the nominal height ratio between the two systems of wrinkles, e.g. $\alpha=5$ in Fig. 4(c). The isotropic honeycomb wrinkle pattern fails to form for loose interactions due to the interference of rippling, Fig. 4(di). Fig. 4(d-ii) shows how an excessive interfacial shear strength relative to the pattern dimensions leads to uncontrolled wrinkles.

\subsection{Local strain at different junction configurations}

Since T-junctions are energetically favorable structures in spontaneous biaxial wrinkling, we turn now to isotropic and anisotropic networks that only exhibit these kinds of junctions, see Fig. 5(a,b). There are many other patterns involving only T-junctions, e.g. chiral patterns obtained from Pythagorean tilings (Grünbaum and Shephard, 1990). We find that for sufficiently tight adhesive interactions ( $h_{0}$ smaller than $\sim 3 \mathrm{~nm}$ ), such patterns form in a very reproducible way, and are not susceptible to instabilities of the junctions. For instance, the rectangular wrinkle pattern in Fig. 5(a) can be achieved for a wide range of strain anisotropies, including isotropic compression. T-junctions are much lower energy configurations as compared to the Y-junctions of the honeycomb lattice, Fig. 5(c). Even though T-junctions are attractive due to their stability, other more energetic junctions may be more interesting from a strain engineering viewpoint. Fig. 5(d) analyzes how strain is distributed spatially. Local in-plane strain has an areal component and a shear component. We focus on the local compressive strain (local area change) as a function of the applied compressive strain $\varepsilon_{C}$ for two types of Y-junctions ( $\mathrm{Y} 1$ and $\mathrm{Y} 2$ ) and a T-junction (T). We quantify the spatial non-uniformity of the local strain by tracking its maximum and minimum values, which occur at the junctions, as well as the local strain in the pla- 

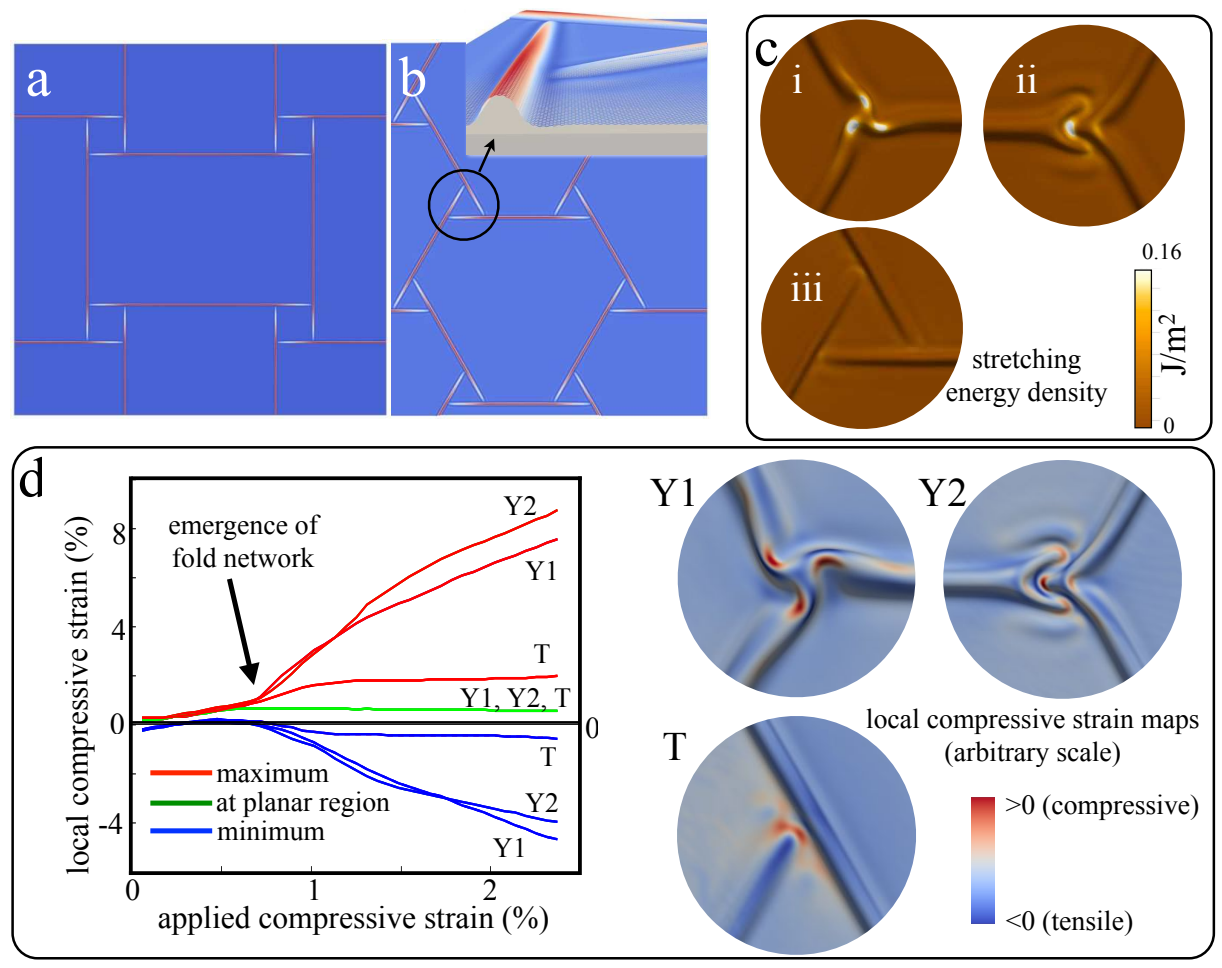

Figure 5: Different junctions types and their local strain behavior. (a) and (b) show variations of the rectangular and honeycomb patterns involving only low-energy T-junctions. (c) Stretching energy of the two types of Y-junction in Fig. 2(b-i) compared to an analogous connecting structure consisting of three T-junctions in (b), at $\varepsilon_{c}=2.4 \%$. (d) Local strain behavior of the two types of Y-junctions and a T-junction as a function of applied strain. The color scale for the local compressive strain maps has been chosen in each case to better show the strain distribution. 



Figure 6: Stability of the wrinkle networks under internal pressure caused by an enclosed fluid. (a-c) and (d,e) show snapshots of Y- and T-junction patterns as mass is added into the interstitial space. The left plot shows the pressure-volume relation along this process for the two patterns, where $V_{0}$ is the volume enclosed by the wrinkled graphene membrane before pressure is applied. Movies of the pressurization processes in (a-c), and (d-f) can be found here [Please insert links to Movies 4 and 5].

nar region away from wrinkles and junctions. T- and Y-junctions exhibit a remarkably different behavior. In patterns with T-junctions, the maximum local strain initially increases as the wrinkle pattern forms, but at later stages remains nearly constant and below the applied compressive strain. For instance, the maximum local strain is about $2 \%$ for $\varepsilon_{C}=2.4 \%$. As for the minimum strain, it remains very small and negative (tensile) throughout the process. This strain attenuation behavior sharply contrasts with that of Y-junctions, which exhibit strong strain amplification that linearly increases with applied strain. For instance, an applied strain of $\varepsilon_{C}=2.4 \%$ results in a local strain ranging between $8 \%$ and $-4 \%$. In all cases, the local strain in the planar regions remains nearly constant and much smaller than the applied strain. Examining the structure of the strain fields, it can be observed that Y-junctions exhibit complex distributions with strongly localized strain dipoles. Thus, it is possible to create wrinkle networks of diverse geometry and junction configurations, which may attenuate or significantly amplify the applied strain, and hence strain-induced changes in properties, at specific points of the network. 


\subsection{Stability of wrinkle networks to interstitial pressure}

Along wrinkles, graphene delaminates from the substrate creating nanochannels. Since graphene is impermeable to most gases and liquids (Bunch et al., 2008; Whitby and Quirke, 2007), wrinkle networks could be harnessed as mechanically self-assembled nano-fluidic devices, possibly in combination with existing methods to encapsulate gas underneath supported graphene (Bunch et al., 2008; Koenig et al., 2011; Zabel et al., 2012). Trapped fluid molecules can create a significant pressure difference across a graphene membrane, and therefore it is important to assess the connectivity and stability of the networks of channels under these conditions.

Following the ideal gas law, we model the effect of gas molecules trapped in the interstitial space between graphene and the substrate by appending the free energy with the term $-n R T \ln \left(V / V_{0}\right)$, where $n$ is the number of moles of gas trapped underneath the membrane, $R$ is the ideal gas constant, $T$ the absolute temperature, and $V_{0}$ an arbitrary reference volume. In simulations, we increase $n$ incrementally, and obtain the pressure $p=n R T / V$ and the volume as part of the analysis. This protocol allows us to follow processes in which the pressure is not monotonic, and is also relevant to some experimental setups (Boddeti et al., 2013). For an enclosed fluid, invoking molecular incompressibility instead of the ideal gas law, this ensemble results in prescribing the enclosed volume.

Fig. 6 shows the response of two isotropic networks, with $\mathrm{Y}$ - and $\mathrm{T}$ junctions, as fluid mass is introduced into the interstitial space until disruption of the network by delamination. We first focus on Y-junctions. We have seen previously (Figs. 4 and 5) that two kinds of Y-junctions can co-exist, but only Y1 provides 3-way connectivity of the channels. As the system becomes pressurized, we observe that all Y2 junctions transform to Y1 junctions. For a wide range of pressures, increasing pressure causes channels to progressively thicken, while maintaining connective junctions, Fig. 6(i). For our choice of adhesion parameters, representative of common substrates, the network can withstand over $80 \mathrm{MPa}$. Previous controlled experiments have created pressure differences across graphene of up to $5 \mathrm{MPa}$. Beyond the maximum pressure, further mass input increases the enclosed volume but decreases the pressure as delamination progresses; triangular blisters nucleate at most junctions, and then some expand while others shrink leading to fewer and very large blisters, see Fig. 6(a-c). These triangular blisters are remarkably similar to those recently observed (Pan et al., 2012). Networks with T-junctions can also withstand high pressure differences by thickening 
the wrinkles as pressure increases. Remarkably, the ends of wrinkles, at Tjunctions, remain sharp in a configuration that blocks fluid flow, Fig. 6(ii). In the post-peak regime, these networks progressively delaminate by widening all the wrinkles away from the junctions, and at a certain point, a few wrinkles quickly grow in volume at the expense of the others, see Fig. 6(d-f). Our simulations confirm the stability of the fixed mass ensemble beyond the onset of delamination, as observed previously in experiments (Boddeti et al., 2013).

In addition to the stability and connectivity of the network, the small dimensions of the channels can pose challenges to nano-fluidic devices transporting liquids. Water diffusing into the interstitial space underneath supported graphene has been shown to freeze at room temperature due to the strong geometric confinement (Lee et al., 2012), as it does inside small carbon nanotubes(Kyakuno et al., 2011). However, if the space inside graphene wrinkles is large enough, water becomes liquid again and flows (Lee et al., 2012). The diameter of the network channels in Fig. 6 is wide enough $(\sim 4$ $\mathrm{nm}$ ) for liquid water, and can be increased either by patterning wider strips of weak adhesion or by increasing the compressive strain.

\section{Summary and conclusions}

We have reproduced computationally the most salient features of wrinkle networks observed in supported graphene. T-junctions, Y-junctions, Xjunctions, and Y-junctions split into three T-junctions similar to those reported here have been observed in CVD graphene samples (Liu et al., 2012). We have identified the influence of strain anisotropy and the adhesive and frictional properties on the morphology of spontaneously-formed wrinkle networks. These results may help interpret or modify CVD growth conditions from the observed wrinkle networks. Our simulations highlight the pivotal role of in-plane stretching of graphene in biaxial wrinkling, which can be interpreted as a process of stretching energy relaxation and focusing at wrinkle junctions.

We have proposed a method to precisely control the location of wrinkles through patterns of stripes of weaker adhesion on the substrate. However, this is only possible within bounds set by the physics of wrinkling. For instance, it is difficult to control wrinkles with very loose adhesive interactions, representative of soft polymeric substrates, unless anisotropic patterns with very different wrinkle heights in each direction are considered. On the other 
hand, the dimensions of the pattern need to be tuned to the friction between graphene and the substrate. By understanding these limitations, we have provided design principles to robustly obtain a variety of network geometries of remarkable regularity, with different wrinkle connectivities and junction configurations. We have shown that T-junction are very stable and attenuate the applied strain, while Y-junctions are strain amplifiers. Finally, we have shown that Y-junction networks may be used in nano-fluidic applications because they provide channel connectivity and are stable up to very high pressure differences. Here, we have limited ourselves to simple periodic networks, but it is possible to create much more general channel topologies, possibly including curved wrinkles.

These ideas may be implemented experimentally by combining strain engineering with nanolithography. Potential applications include mechanically self-assembled nano-fluidic devices, or local and reversible modification of the electronic, chemical or optical properties of graphene by curvature or self-adhesion along lines (wrinkles) or by stretch at points (junctions). The ideas presented here are applicable to any elastic thin film weakly bonded to a substrate, including multilayer graphene or other atomically thin crystalline materials such as $\mathrm{MoS}_{2}$ (Castellanos-Gomez et al., 2013).

\section{Acknowledgement}

We acknowledge the support of the European Research Council (FP7/20072013)/ERC grant Agreement No. 240487.

\section{Appendix A. Sensitivity to numerical parameters}

We examine here the sensitivity of the computational result to key numerical parameters: mesh refinement, load increment size, and dimensions of the periodic domain. We performed many simulations varying these parameters within the computationally feasible range, some of which are reported in Fig. A.7. We found that the precise location of wrinkles is completely different when the load increment size or the dimensions of the periodic box are changed (Fig. A.7(a)). This variability is a consequence of the massive non-uniqueness of equilibrium states in the system. However, the general features of the pattern, in terms of wrinkle spacing and orientations, are similar. More quantitatively, as shown by the evolution of the different energy components as a function of strain in Fig. A.7(b), the numerical equilibria 
for different computational parameters exhibit very similar global behavior. Our simulations appear to be converged in terms of the periodic domain and mesh size, and the most noticeable differences are observed for large load steps, with a delayed ripple to wrinkle transition, presumably due to an inaccurate estimation of the work of friction. In summary, given the fundamental metastability of buckling in highly symmetric systems, our results are remarkably insensitive to the key numerical parameters.

\section{References}

Aitken, Z.H., Huang, R., 2010. Effects of mismatch strain and substrate surface corrugation on morphology of supported monolayer graphene. Journal of Applied Physics 107, 123531.

Aoyanagi, Y., Hure, J., Bico, J., Roman, B., 2010. Random blisters on stickers: metrology through defects. Soft Matter 6, 5720-5728.

Arias, I., Arroyo, M., 2008. Size-dependent nonlinear elastic scaling of multiwalled carbon nanotubes. Phys. Rev. Lett. 100, 085503.

Arroyo, M., Belytschko, T., 2002. An atomistic-based finite deformation membrane for single layer crystalline films. Journal of the Mechanics and Physics of Solids 50, 1941-1977.

Arroyo, M., Belytschko, T., 2004a. Finite crystal elasticity of carbon nanotubes based on the exponential cauchy-born rule. Physical Review B 69, 115415 .

Arroyo, M., Belytschko, T., 2004b. Finite element methods for the non-linear mechanics of crystalline sheets and nanotubes. Int. J. Numer. Meth. Engng $59,419-456$.

Boddeti, N.G., Liu, X., Long, R., Xiao, J., Bunch, J.S., Dunn, M.L., 2013. Graphene blisters with switchable shapes controlled by pressure and adhesion. Nano Letters 13, 6216-6221.

Bunch, J.S., Verbridge, S.S., Alden, J.S., van der Zande, A.M., Parpia, J.M., Craighead, H.G., McEuen, P.L., 2008. Impermeable atomic membranes from graphene sheets. Nano Letters 8, 2458-2462. 

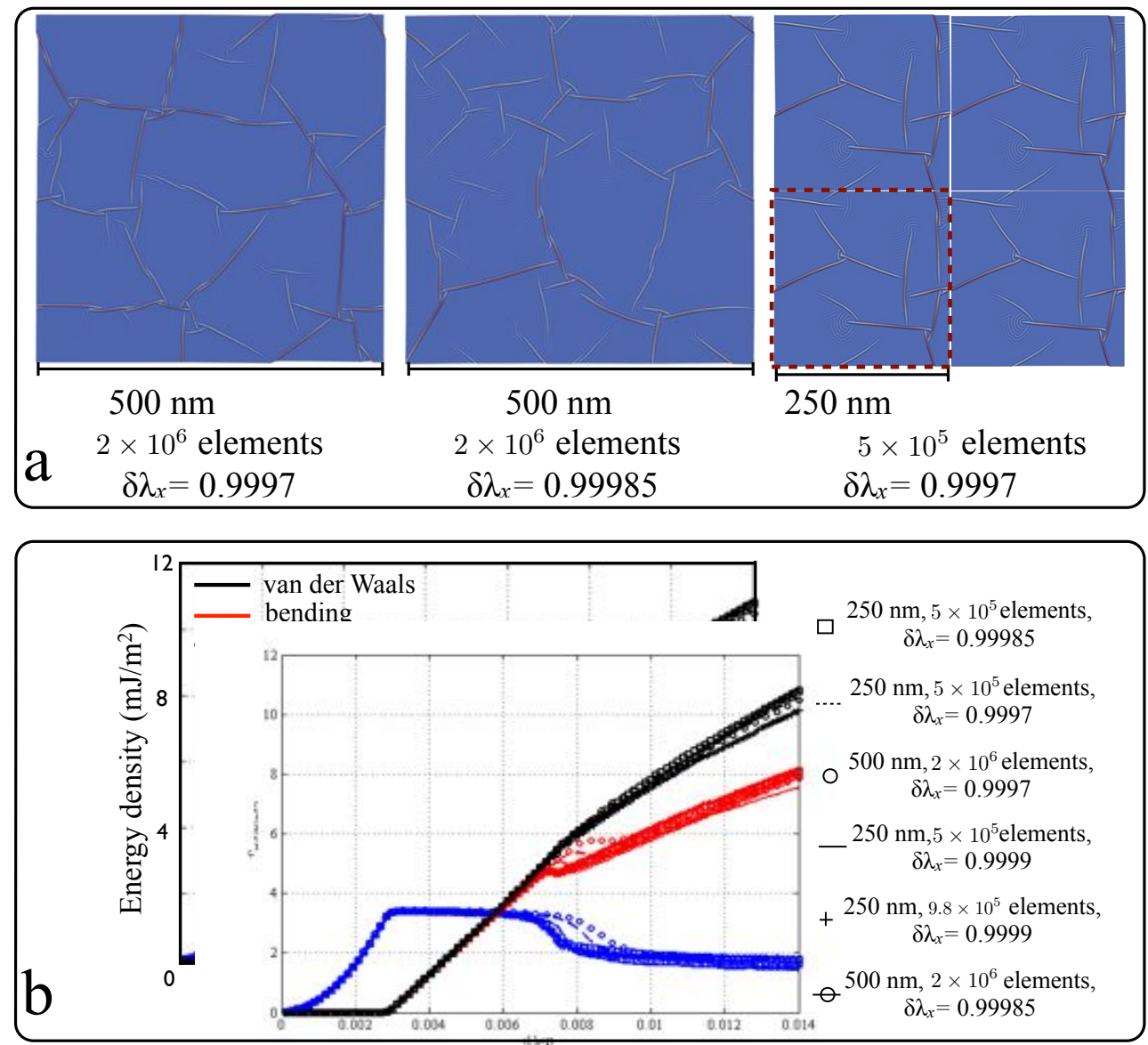

Figure A.7: Wrinkling pattern (a) and different contributions to the energy of the system as a function of applied strain (b), as the key numerical parameters - mesh size, load increment size, and dimensions of the periodic domain - are varied. In all the simulations, $\tau_{0}=1.8 \mathrm{MPa}, h_{0}=1.5 \mathrm{~nm}, \gamma=0.45 \mathrm{~J} / \mathrm{m}^{2}$. 
Calado, V.E., Schneider, G.F., Theulings, A.M.M.G., Dekker, C., Vandersypen, L.M.K., 2012. Formation and control of wrinkles in graphene by the wedging transfer method. Applied Physics Letters 101, 103116.

Castellanos-Gomez, A., Roldán, R., Cappelluti, E., Buscema, M., Guinea, F., van der Zant, H.S.J., Steele, G.A., 2013. Local strain engineering in atomically thin mos2. Nano Letters doi: 10.1021/nl402875m.

Cerda, E., Chaieb, S., Melo, F., Mahadevan, L., 1999. Conical dislocations in crumpling. Nature 401, 46-49.

Duong, D.L., Han, G.H., Lee, S.M., Gunes, F., Kim, E.S., Kim, S.T., Kim, H., Ta, Q.H., So, K.P., Yoon, S.J., Chae, S.J., Jo, Y.W., Park, M.H., Chae, S.H., Lim, S.C., Choi, J.Y., Lee, Y.H., 2012. Probing graphene grain boundaries with optical microscopy. Nature 490, 235-239.

Gao, W., Huang, R., 2011. Effect of surface roughness on adhesion of graphene membranes. Journal of Physics D: Applied Physics 44, 452001.

Garcia-Pomar, J.L., Nikitin, A.Y., Martin-Moreno, L., 2013. Scattering of graphene plasmons by defects in the graphene sheet. ACS Nano 7, 49884994.

Georgiou, T., Britnell, L., Blake, P., Gorbachev, R.V., Gholinia, A., Geim, A.K., Casiraghi, C., Novoselov, K.S., 2011. Graphene bubbles with controllable curvature. Applied Physics Letters 99, 093103.

Ghosh, S., Arroyo, M., 2013. An atomistic-based foliation model for multilayer graphene materials and nanotubes. Journal of the Mechanics and Physics of Solids 61, $235-253$.

Grünbaum, B., Shephard, G.C., 1990. Tilings and Patterns. W.H. Freeman.

Gupta, A., Chen, G., Joshi, P., Tadigadapa, S., Eklund, 2006. Raman scattering from high-frequency phonons in supported n-graphene layer films. Nano Letters 6, 2667-2673.

Hendricks, T.R., Wang, W., Lee, I., 2010. Buckling in nanomechanical films. Soft Matter 6, 3701-3706. 
Huang, Z.Y., Hong, W., Suo, Z., 2005. Nonlinear analyses of wrinkles in a film bonded to a compliant substrate. Journal of the Mechanics and Physics of Solids 53, 2101-2118.

Jiang, T., Huang, R., Zhu, Y., 2013. Interfacial sliding and buckling of monolayer graphene on a stretchable substrate. Advanced Functional Materials 24, 396-402.

Kim, K., Lee, Z., Malone, B.D., Chan, K.T., Alemán, B., Regan, W., Gannett, W., Crommie, M.F., Cohen, M.L., Zettl, A., 2011a. Multiply folded graphene. Phys. Rev. B 83, 245433.

Kim, P., Abkarian, M., Stone, H.A., 2011b. Hierarchical folding of elastic membranes under biaxial compressive stress. Nat Mater 10, 952-957.

Kitt, A.L., Qi, Z., R@mi, S., Park, H.S., Swan, A.K., Goldberg, B.B., 2013. How graphene slides: Measurement and theory of strain-dependent frictional forces between graphene and sio2. Nano Letters 13, 2605-2610.

Koenig, S.P., Boddeti, N.G., Dunn, M.L., Bunch, J.S., 2011. Ultrastrong adhesion of graphene membranes. Nat Nano 6, 543-546.

Kyakuno, H., Matsuda, K., Yahiro, H., Inami, Y., Fukuoka, T., Miyata, Y., Yanagi, K., Maniwa, Y., Kataura, H., Saito, T., Yumura, M., Iijima, S., 2011. Confined water inside single-walled carbon nanotubes: Global phase diagram and effect of finite length. The Journal of Chemical Physics 134, 244501.

Lee, M., Choi, J., Kim, J.S., Byun, I.S., Lee, D., Ryu, S., Lee, C., Park, B., 2012. Characteristics and effects of diffused water between graphene and a sio2 substrate. Nano Research 5, 710-717.

Levy, N., Burke, S.A., Meaker, K.L., Panlasigui, M., Zettl, A., Guinea, F., Neto, A.H.C., Crommie, M.F., 2010. Strain-induced pseudo-magnetic fields greater than 300 tesla in graphene nanobubbles. Science 329, 544547.

Li, X., Cai, W., An, J., Kim, S., Nah, J., Yang, D., Piner, R., Velamakanni, A., Jung, I., Tutuc, E., Banerjee, S.K., Colombo, L., Ruoff, R.S., 2009. Large-area synthesis of high-quality and uniform graphene films on copper foils. Science 324, 1312-1314. 
Liu, M., Zhang, Y., Chen, Y., Gao, Y., Gao, T., Ma, D., Ji, Q., Zhang, Y., Li, C., Liu, Z., 2012. Thinning segregated graphene layers on high carbon solubility substrates of rhodium foils by tuning the quenching process. ACS Nano 6, 10581-10589.

Liu, N., Pan, Z., Fu, L., Zhang, C., Dai, B., Liu, Z., 2011. The origin of wrinkles on transferred graphene. Nano Research 4, 996-1004.

Liu, Y., Yakobson, B.I., 2010. Cones, pringles, and grain boundary landscapes in graphene topology. Nano Letters 10, 2178-2183.

Lu, Q., Arroyo, M., Huang, R., 2009. Elastic bending modulus of monolayer graphene. Journal of Physics D: Applied Physics 42, 102002.

Obraztsov, A.N., Obraztsova, E.A., Tyurnina, A.V., Zolotukhin, A.A., 2007. Chemical vapor deposition of thin graphite films of nanometer thickness. Carbon 45, 2017-2021.

Pan, W., Xiao, J., Zhu, J., Yu, C., Zhang, G., Ni, Z., Watanabe, K., Taniguchi, T., Shi, Y., Wang, X., 2012. Biaxial compressive strain engineering in graphene/boron nitride heterostructures. Sci. Rep. 2, 893.

Pan, Z., Liu, N., Fu, L., Liu, Z., 2011. Wrinkle engineering: A new approach to massive graphene nanoribbon arrays. Journal of the American Chemical Society 133, 17578-17581.

Pereira, V.M., Castro Neto, A.H., Liang, H.Y., Mahadevan, L., 2010. Geometry, mechanics, and electronics of singular structures and wrinkles in graphene. Phys. Rev. Lett. 105, 156603.

Pocivavsek, L., Dellsy, R., Kern, A., Johnson, S., Lin, B., Lee, K.Y.C., Cerda, E., 2008. Stress and fold localization in thin elastic membranes. Science $320,912-916$.

Pugno, N., Yin, Q., Shi, X., Capozza, R., 2013. A generalization of the coulomb friction law: from graphene to macroscale. Meccanica 48, 18451851.

Robertson, A.W., Bachmatiuk, A., Wu, Y.A., Schäffel, F., Büchner, B., Rümmeli, M.H., Warner, J.H., 2011. Structural distortions in few-layer graphene creases. ACS Nano 5, 9984-9991. 
Ruoff, R., 2012. Perspective: A means to an end. Nature 483, S42.

Scharfenberg, S., Mansukhani, N., Chialvo, C., Weaver, R.L., Mason, N., 2012. Observation of a snap-through instability in graphene. Applied Physics Letters 100, 021910.

Srivastava, D., Brenner, D.W., Schall, J.D., Ausman, K.D., Yu, M., Ruoff, R.S., 1999. Predictions of enhanced chemical reactivity at regions of local conformational strain on carbon nanotubes: Kinky chemistry. The Journal of Physical Chemistry B 103, 4330-4337.

Stolyarova, E., Stolyarov, D., Bolotin, K., Ryu, S., Liu, L., Rim, K.T., Klima, M., Hybertsen, M., Pogorelsky, I., Pavlishin, I., Kusche, K., Hone, J., Kim, P., Stormer, H.L., Yakimenko, V., Flynn, G., 2009. Observation of graphene bubbles and effective mass transport under graphene films. Nano Letters 9, 332-337.

Vandeparre, H., Léopoldès, J., Poulard, C., Desprez, S., Derue, G., Gay, C., Damman, P., 2007. Slippery or sticky boundary conditions: Control of wrinkling in metal-capped thin polymer films by selective adhesion to substrates. Phys. Rev. Lett. 99, 188302.

Vella, D., Bico, J., Boudaoud, A., Roman, B., Reis, P.M., 2009. The macroscopic delamination of thin films from elastic substrates. Proceedings of the National Academy of Sciences 106, 10901-10906.

Wang, Y., Yang, R., Shi, Z., Zhang, L., Shi, D., Wang, E., Zhang, G., 2011. Super-elastic graphene ripples for flexible strain sensors. ACS Nano 5, 3645-3650.

Warner, J.H., Fan, Y., Robertson, A.W., He, K., Yoon, E., Lee, G.D., 2013. Rippling graphene at the nanoscale through dislocation addition. Nano Letters 13, 4937-4944.

Whitby, M., Quirke, N., 2007. Fluid flow in carbon nanotubes and nanopipes. Nat Nano 2, 87-94.

Witten, T.A., 2007. Stress focusing in elastic sheets. Rev. Mod. Phys. 79, 643-675. 
Xu, K., Cao, P., Heath, J.R., 2009. Scanning tunneling microscopy characterization of the electrical properties of wrinkles in exfoliated graphene monolayers. Nano Letters 9, 4446-4451.

Yakobson, B.I., Ding, F., 2011. Observational geology of graphene, at the nanoscale. ACS Nano 5, 1569-1574.

Yoon, T., Shin, W.C., Kim, T.Y., Mun, J.H., Kim, T.S., Cho, B.J., 2012. Direct measurement of adhesion energy of monolayer graphene as-grown on copper and its application to renewable transfer process. Nano Letters $12,1448-1452$.

Zabel, J., Nair, R.R., Ott, A., Georgiou, T., Geim, A.K., Novoselov, K.S., Casiraghi, C., 2012. Raman spectroscopy of graphene and bilayer under biaxial strain: Bubbles and balloons. Nano Letters 12, 617-621.

Zang, J., Ryu, S., Pugno, N., Wang, Q., Tu, Q., Buehler, M.J., Zhao, X., 2013. Multifunctionality and control of the crumpling and unfolding of large-area graphene. Nature Materials 12, 321-325.

Zang, J., Zhao, X., Cao, Y., Hutchinson, J.W., 2012. Localized ridge wrinkling of stiff films on compliant substrates. Journal of the Mechanics and Physics of Solids 60, 1265-1279.

Zhang, K., Arroyo, M., 2013. Adhesion and friction control localized folding in supported graphene. Journal of Applied Physics 113, 193501.

Zhang, T., Li, X., Gao, H., 2014. Defects controlled wrinkling and topological design in graphene. Journal of the Mechanics and Physics of Solids 67, 2 -13 .

Zhu, W., Low, T., Perebeinos, V., Bol, A.A., Zhu, Y., Yan, H., Tersoff, J., Avouris, P., 2012. Structure and electronic transport in graphene wrinkles. Nano Letters 12, 3431-3436. 